\title{
ANNULAR ITINERARIES FOR ENTIRE FUNCTIONS
}

\author{
P. J. RIPPON AND G. M. STALLARD
}

Abstract. In order to analyse the way in which the size of the iterates of a transcendental entire function $f$ can behave, we introduce the concept of the annular itinerary of a point $z$. This is the sequence of non-negative integers $s_{0} s_{1} \ldots$ defined by

$$
f^{n}(z) \in A_{s_{n}}(R), \text { for } n \geq 0,
$$

where $A_{0}(R)=\{z:|z|<R\}$ and

$$
A_{n}(R)=\left\{z: M^{n-1}(R) \leq|z|<M^{n}(R)\right\}, \quad n \geq 1 .
$$

Here $M(r)$ is the maximum modulus of $f$ on $\{z:|z|=r\}$ and $R>0$ is so large that $M(r)>r$, for $r \geq R$.

We consider the different types of annular itineraries that can occur for any transcendental entire function $f$ and show that it is always possible to find points with various types of prescribed annular itineraries. The proofs use two new annuli covering results that are of wider interest.

\section{INTRODUCTION}

Let $f: \mathbb{C} \rightarrow \mathbb{C}$ be a transcendental entire function and denote by $f^{n}, n=$ $0,1,2, \ldots$, the $n$th iterate of $f$. The Fatou set $F(f)$ is the set of points $z \in \mathbb{C}$ such that $\left(f^{n}\right)_{n \in \mathbb{N}}$ forms a normal family in some neighborhood of $z$. The complement of $F(f)$ is called the Julia set $J(f)$ of $f$. The escaping set $I(f)$ of $f$ is defined to be

$$
I(f)=\left\{z: f^{n}(z) \rightarrow \infty \text { as } n \rightarrow \infty\right\},
$$

and it is known [7] that we always have $J(f)=\partial I(f)$. An introduction to the properties of these sets can be found in [2].

One technique that has often been used to understand the nature of the above sets is 'symbolic dynamics'. After partitioning $\mathbb{C}$ in some manner and labelling each subset in the partition by a different symbol, we obtain the 'itinerary' of a point $z \in \mathbb{C}$ by taking the sequence of symbols corresponding to the successive subsets of the partition visited by the iterates $\left(f^{n}(z)\right)$ of $z$.

This technique was used, for example, in [6] to show that, for certain entire functions, the sets $J(f)$ and $I(f)$ contain so-called 'Cantor bouquets' of curves, by studying those points with common itineraries with respect to certain natural partitions. This approach was developed and refined in [17] to prove that for a large class of entire functions, including all functions of finite order in the much-studied Eremenko-Lyubich class $\mathcal{B}$ (see [8]), all points of $I(f)$ can be joined to $\infty$ by a curve in $I(f)$, a result that is related to a conjecture of Eremenko in [7].

Received by the editors January 24, 2013.

2010 Mathematics Subject Classification. Primary 37F10; Secondary 30D05.

Both authors were supported by the EPSRC grant EP/H006591/1. 
Symbolic dynamics was also used in [12] in order to analyse the dynamical properties of the components of the complement of the fast escaping set $A(f)$ for functions $f$ such that the set $A_{R}(f)$ has the structure of a 'spider's web', in particular, to show that in this case there are uncountably many components of $A(f)^{c}$ of various types. Here

$$
A_{R}(f)=\left\{z:\left|f^{n}(z)\right| \geq M^{n}(R), \text { for } n \in \mathbb{N}\right\} \quad \text { and } \quad A(f)=\bigcup_{\ell \in \mathbb{N}} f^{-\ell}\left(A_{R}(f)\right),
$$

where $M(r)=M(r, f)=\max _{|z|=r}|f(z)|, r>0, M^{n}(r)$ denotes iteration of $M(r)$, and $R>0$ is any value such that $M(r)>r$ for $r \geq R$. See [15] for the detailed properties of $A(f)$ and the many families of functions for which $A_{R}(f)$ is a spider's web. Note that this spider's web structure cannot occur for functions in the class $\mathcal{B}$, so the results in [12] are complementary to those in [6] and [17].

Here we introduce a new type of partition of the plane which is appropriate for analysing a key aspect of the dynamical behaviour of any transcendental entire function $f$, namely, the possible ways in which the size of the iterates $f^{n}(z), n \geq 0$, can vary. We define this partition as follows: $A_{0}(R)=\{z:|z|<R\}$ and

$$
A_{n}(R)=\left\{z: M^{n-1}(R) \leq|z|<M^{n}(R)\right\}, \quad n \geq 1,
$$

where $R>0$ is any value such that $M(r)>r$ for $r \geq R$. Then, for any $z \in \mathbb{C}$, the sequence of non-negative integers $s_{0} s_{1} \ldots$ defined by

$$
f^{n}(z) \in A_{s_{n}}(R), \text { for } n \geq 0,
$$

will be called the annular itinerary of $z$, with respect to the partition $\left\{A_{n}(R)\right\}$.

Note that for any annular itinerary $s_{0} s_{1} \ldots$ we have $s_{n+1} \leq s_{n}+1$ for $n \geq 0$ by the maximum principle. Also, if the value $R$ changes to $R^{\prime}$, then the annular itinerary, $s_{0} s_{1} \ldots$ say, of a point changes to $s_{0}^{\prime} s_{1}^{\prime} \ldots$ where, for some integer $p$,

$$
s_{n}^{\prime}-s_{n} \in\{p, p+1\}, \text { for } n \geq 0 .
$$

Our first result enables us to construct points with many different types of annular itineraries.

Theorem 1.1. Let $f$ be a transcendental entire function. There exists $R=R(f)>0$ and a sequence of closed annuli

$$
B_{n}=\left\{z: r_{n} \leq|z| \leq r_{n}^{\prime}\right\}, \quad n \geq 0,
$$

each of which meets $J(f)$, such that

$$
\begin{gathered}
B_{n} \subset A_{n}(R), \text { for } n \geq 0, \\
f\left(B_{n}\right) \supset B_{n+1}, \text { for } n \geq 0,
\end{gathered}
$$

and there is a subsequence $B_{n_{j}}$ such that, for $j \in \mathbb{N}$,

$$
f\left(B_{n_{j}}\right) \supset B_{n}, \text { for } 0 \leq n \leq n_{j} \text {, with at most one exception. }
$$

Moreover, if $f$ has a multiply connected Fatou component, then the annuli $B_{n}$ can be chosen in such a way that (1.4) holds with no exceptions and the sequence $\left(n_{j}\right)$ consists of those $n \geq 0$ such that $A_{n}(R)$ contains a zero of $f$.

Remark. It is clear that the value $R=R(f)$ in Theorem 1.1 can be chosen to be arbitrarily large. 
Note that any multiply connected Fatou component $U$ of $f$ is an escaping wandering domain whose forward images form a sequence of eventually nested 'ring-like' domains; see Lemma 3.1

Theorem 1.1 gives the following sufficient condition for a sequence $s_{0} s_{1} \ldots$ to be an annular itinerary.

Theorem 1.2. Let $f$ be a transcendental entire function. There exist $R=R(f)>0$, a sequence $n_{j}, j \in \mathbb{N}$, of positive integers with $n_{j} \rightarrow \infty$ as $j \rightarrow \infty$, and a sequence of sets $I_{j}, j \in \mathbb{N}$, where $I_{j} \subset\left\{0, \ldots, n_{j}\right\}$ has at most one element, such that:

if $s_{0} s_{1} \ldots$ is any sequence of non-negative integers with the property that

$$
\begin{aligned}
& \text { for } m \geq 0, \text { we have } s_{m+1}=s_{m}+1 \text { or, in the case that } s_{m}=n_{j} \text { for } \\
& \text { some } j \in \mathbb{N}, s_{m+1} \in\left\{0, \ldots, n_{j}\right\} \backslash I_{j},
\end{aligned}
$$

then there exists $\zeta \in J(f)$ with annular itinerary $s_{0} s_{1} \ldots$ with respect to $\left\{A_{n}(R)\right\}$.

Moreover, if $f$ has a multiply connected Fatou component, then $I_{j}=\emptyset$, for $j \in \mathbb{N}$, and the sequence $\left(n_{j}\right)$ consists of those $n \geq 0$ such that $A_{n}(R)$ contains a zero of $f$.

Theorem [1.2 is related to results in [12, Theorem 1.2], where it is assumed that $A_{R}(f)$ is a spider's web. It implies that for any transcendental entire function there exist many types of prescribed annular itineraries, including those in the following result. Note that a version of Corollary 1.3 part (d) was proved in [14, Theorem 1].

Corollary 1.3. Let $f$ be a transcendental entire function, let $R=R(f)>0$ be the value given by Theorem 1.2 and let $s \in \mathbb{N}$. Then there exist points with the following types of annular itineraries $\left(s_{n}\right)$ with respect to $\left\{A_{n}(R)\right\}$ :

(a) periodic itineraries of all periods with at most one exception such that $s_{n} \geq s$ for $n \geq 0$;

(b) uncountably many itineraries such that $\left(s_{n}\right)$ is bounded and $s_{n} \geq s$ for $n \geq 0$

(c) uncountably many itineraries such that $\left(s_{n}\right)$ is unbounded but does not tend to $\infty$, and $s_{n} \geq s$ for $n \geq 0$;

(d) uncountably many itineraries such that $s_{n} \rightarrow \infty$ as $n \rightarrow \infty$ more slowly than at any given rate.

By definition a point is in $A(f)$ if and only if its itinerary $s_{0} s_{1} \ldots$ with respect to any $\left\{A_{n}(R)\right\}$ satisfies $s_{n+1}=s_{n}+1$ for sufficiently large $n$. The existence of such points is well known (see [3] or [15]) and also follows from Theorem 1.2. There are clearly only countably many such itineraries. On the other hand, it follows from our final result that for any given rate of escape that is less than that of 'fast escape' there exist uncountably many itineraries for which the corresponding points escape at least at this given rate and in some sense at no faster rate.

Theorem 1.4. Let $f$ be a transcendental entire function. There exists $R=R(f)>0$ with the property that whenever $\left(a_{n}\right)$ is a positive sequence such that

$$
a_{n} \geq R \text { and } a_{n+1} \leq M\left(a_{n}\right), \text { for } n \geq 0,
$$

there exist a point $\zeta \in J(f)$ and a sequence $\left(n_{j}\right)$ with $n_{j} \rightarrow \infty$ as $j \rightarrow \infty$ such that

$$
\left|f^{n}(\zeta)\right| \geq a_{n}, \text { for } n \geq 0, \text { and }\left|f^{n_{j}}(\zeta)\right| \leq M^{2}\left(a_{n_{j}}\right) \text {, for } j \in \mathbb{N} .
$$

If, in addition,

$$
\text { for each } \ell \in \mathbb{N} \text { there exists } n(\ell) \in \mathbb{N} \text { such that } a_{n(\ell)+\ell}<M^{n(\ell)}(R),
$$


then there are uncountably many itineraries with respect to $\left\{A_{n}(R)\right\}$ that correspond to points $\zeta$ satisfying (1.7).

Remarks. 1. In Theorem 1.4 we cannot replace (1.7) by

$$
a_{n} \leq\left|f^{n}(\zeta)\right| \leq C a_{n}, \quad \text { for sufficiently large } n,
$$

where $C>1$ is an absolute constant. Indeed, [14, proof of Theorem 2] shows that if there exist $\zeta \in J(f)$ and $C>1$ such that (1.9) holds whenever $\left(a_{n}\right)$ is a positive sequence such that $a_{n} \rightarrow \infty$ as $n \rightarrow \infty$ and $a_{n+1} \leq M\left(a_{n}\right)$ for $n \in \mathbb{N}$, then there must exist constants $c>0, d>1$ and $r_{0}>0$ such that

$$
\text { for all } r \geq r_{0} \text { we have } m(s) \leq c \text { for some } s \in(r, d r),
$$

where $m(r)$ denotes the minimum modulus of $f$ :

$$
m(r)=m(r, f)=\min _{|z|=r}|f(z)|, \quad \text { for } r>0 .
$$

There are many transcendental entire functions that do not satisfy (1.10), in particular those with a multiply connected Fatou component.

2. In [16] we use Theorem 1.4 to give a necessary and sufficient condition for a certain subset of $I(f)$ called $Q(f)$, the 'quite fast escaping set', to equal $A(f)$. An important special case of the results in [16] is that $Q(f)=A(f)$ for functions in the class $\mathcal{B}$.

We prove the results above by using a method that has its origins in one that we introduced in [14] in order to construct points that escape arbitrarily slowly. This method involves two complementary annuli covering results which are significant generalisations of results in [14] and of independent interest. Both results are given in Section 2

In Section 3 we prove a version of Theorem 1.1 for a transcendental entire function that has a multiply connected Fatou component, and in Section 4 we prove a version for a transcendental entire function that has no multiply connected Fatou components. The proofs in these two cases are significantly different. In Section 5 we prove Theorem 1.2 and Corollary 1.3, and in Section 6 we prove versions of Theorem 1.4 in each of the two cases mentioned above, showing that we obtain a much better upper estimate than the one in (1.7) when there are no multiply connected Fatou components.

\section{Annuli covering theorems}

To obtain the sequence of annuli in Theorem 1.1 we prove two theorems about covering properties of annuli, which are considerably stronger than previous results of this nature. Here and later in the paper we use the following basic facts about the maximum modulus.

Lemma 2.1. Let $f$ be a transcendental entire function. Then

(a) $\frac{\log M(r)}{\log r} \rightarrow \infty$ as $r \rightarrow \infty$;

(b) there exists $R_{0}=R_{0}(f)>0$ such that

$$
M\left(r^{k}\right) \geq M(r)^{k}, \text { for } r \geq R_{0}, k>1 ;
$$

(c) $\frac{M(2 r)}{M(r)} \rightarrow \infty$ as $r \rightarrow \infty$. 
Part (a) is a standard property of a transcendental entire function and part (b) is a version of Hadamard convexity (see [13, Lemma 2.1]). Part (c) is well known and easily follows from part (b), for example.

Our first covering theorem is of a similar nature to [14, Lemma 3], but the conclusion is much stronger and the proof is simpler, since it uses the contraction property of the hyperbolic metric in a similar way to the proof of [4, Theorem 3.3]. We denote the density of the hyperbolic metric at a point $z$ in a hyperbolic domain $G$ by $\rho_{G}(z)$ and the hyperbolic distance between $z_{1}$ and $z_{2}$ in $G$ by $\rho_{G}\left(z_{1}, z_{2}\right)$. Also, for $w \in \mathbb{C}$ and $0<r<s$ we write

$$
\begin{gathered}
B(w, r)=\{z:|z-w|<r\} \\
A(r, s)=\{z: r<|z|<s\} \text { and } \bar{A}(r, s)=\{z: r \leq|z| \leq s\},
\end{gathered}
$$

and we recall that the minimum modulus $m(r)=m(r, f)$ was defined in (1.10).

Theorem 2.2. Let $f$ be a transcendental entire function. There exists $R_{1}=$ $R_{1}(f)>0$ such that if $r \geq R_{1}$ and

$$
\text { there exists } s \in(2 r, 4 r) \text { such that } m(s) \leq 1 \text {, }
$$

then either

$$
f(A(r, 8 r)) \supset B(0, M(r))
$$

or there exists $w_{1} \in B(0, M(r))$ such that $f$ omits $w_{1}$ in $A(r, 8 r)$ and

$$
f(A(r, 8 r)) \supset B(0, M(r)) \backslash B\left(w_{1}, \varepsilon(r) \max \left\{\left|w_{1}\right|, 1\right\}\right),
$$

where

$$
\varepsilon(r)=\frac{c_{1}}{\lambda(r)^{c_{0}}}, \text { with } \lambda(r)=\frac{1}{2}\left(\frac{M(2 r)}{M(r)}-1\right),
$$

and $c_{0}, c_{1}$ are absolute constants such that $0<c_{0}<1$ and $c_{1}>2$.

Note that $\varepsilon(r) \rightarrow 0$ as $r \rightarrow \infty$, by Lemma 2.1 part (c), so we have the following corollary of Theorem 2.2 .

Corollary 2.3. Let $f$ be a transcendental entire function. There exists $R_{2}=$ $R_{2}(f)>0$ such that if $r \geq R_{2}$, (2.1) holds, and $S, S^{\prime}, T, T^{\prime}$ satisfy

$$
2<S<S^{\prime}, T<T^{\prime} \leq M(r) \text { and } S^{\prime} \leq \frac{1}{2} T
$$

then

$$
f(A(r, 8 r)) \text { contains } A\left(S, S^{\prime}\right) \text { or } A\left(T, T^{\prime}\right) \text {. }
$$

Proof of Theorem 2.2. We initially take $R_{1}=R_{1}(f)>0$ so large that $\lambda(r) \geq 2$, for $r \geq R_{1}$, and suppose that (2.1) holds for some $r \geq R_{1}$. Clearly, $A(r, 8 r) \supset A\left(\frac{1}{2} s, 2 s\right)$. Then take $\zeta$ and $\zeta^{\prime}$ such that $|\zeta|=\left|\zeta^{\prime}\right|=s$, and

$$
|f(\zeta)| \leq 1 \text { and }\left|f\left(\zeta^{\prime}\right)\right|=M(s) \geq M(2 r) .
$$

There is an absolute constant $C_{0}>1$ such that $\rho_{A(s / 2,2 s)}\left(\zeta, \zeta^{\prime}\right) \leq \frac{1}{2} \log C_{0}$.

Suppose that $f$ omits in $A(s / 2,2 s)$ two values $w_{1}, w_{2} \in B(0, M(r))$ such that

$$
\left|w_{2}-w_{1}\right|=\beta \max \left\{\left|w_{1}\right|, 1\right\} \text {, where } \beta>0 .
$$

We need to show that $\beta<\varepsilon(r)$, where $\varepsilon(r)$ is of the form given in (2.3). 
By Pick's theorem [5, Theorem 4.1],

$$
\begin{aligned}
\frac{1}{2} \log C_{0} & \geq \rho_{A(s / 2,2 s)}\left(\zeta, \zeta^{\prime}\right) \\
& \geq \rho_{f(A(s / 2,2 s))}\left(f(\zeta), f\left(\zeta^{\prime}\right)\right) \\
& >\rho_{\mathbb{C} \backslash\left\{w_{1}, w_{2}\right\}}\left(f(\zeta), f\left(\zeta^{\prime}\right)\right) \\
& =\rho_{\mathbb{C} \backslash\{0,1\}}\left(L(f(\zeta)), L\left(f\left(\zeta^{\prime}\right)\right)\right),
\end{aligned}
$$

where $L(w)=\left(w-w_{1}\right) /\left(w_{2}-w_{1}\right)$. Then, by (2.5) and (2.6),

and

$$
|L(f(\zeta))| \leq \frac{|f(\zeta)|+\left|w_{1}\right|}{\left|w_{2}-w_{1}\right|} \leq \frac{1+\left|w_{1}\right|}{\beta \max \left\{\left|w_{1}\right|, 1\right\}} \leq \frac{2}{\beta}
$$

$$
\left|L\left(f\left(\zeta^{\prime}\right)\right)\right| \geq \frac{\left|f\left(\zeta^{\prime}\right)\right|-\left|w_{1}\right|}{\left|w_{2}\right|+\left|w_{1}\right|} \geq \frac{M(2 r)-M(r)}{2 M(r)}=\frac{1}{2}\left(\frac{M(2 r)}{M(r)}-1\right)=\lambda(r) .
$$

We can assume that $\lambda(r)>2 / \beta$, since otherwise $\beta \leq 2 / \lambda(r)<c_{1} / \lambda(r)^{c_{0}}$ whenever $0<c_{0}<1$ and $c_{1}>2$. If we also have $2 / \beta \geq 2$, then, by (2.7) and [9, Theorem 9.14],

$$
\begin{aligned}
\frac{1}{2} \log C_{0} & >\rho_{\mathbb{C} \backslash\{0,1\}}\left(L(f(\zeta)), L\left(f\left(\zeta^{\prime}\right)\right)\right) \\
& \geq \int_{2 / \beta}^{\lambda(r)} \frac{d t}{2 t \log \left(C_{1} t\right)}=\frac{1}{2} \log \frac{\log \left(C_{1} \lambda(r)\right)}{\log \left(2 C_{1} / \beta\right)},
\end{aligned}
$$

where $C_{1}>1$ is an absolute constant, and hence

$$
\beta<\frac{2 C_{1}}{\left(C_{1} \lambda(r)\right)^{1 / C_{0}}}=\frac{c_{1}}{\lambda(r)^{c_{0}}},
$$

where $c_{0}=1 / C_{0} \in(0,1)$ and $c_{1}=2 C_{1}^{1-1 / C_{0}}>2$, as required.

If, on the other hand, we have $2 / \beta<2$, then the reasoning in (2.8) gives

$$
\frac{1}{2} \log C_{0}>\int_{2}^{\lambda(r)} \frac{d t}{2 t \log \left(C_{1} t\right)}=\frac{1}{2} \log \frac{\log \left(C_{1} \lambda(r)\right)}{\log \left(2 C_{1}\right)},
$$

which is impossible if $R_{1}(f)$ is so large that $\lambda(r) \geq\left(2 C_{1}\right)^{C_{0}} / C_{1}$ for $r \geq R_{1}(f)$.

Our second covering theorem is complementary to Theorem 2.2, since we assume here that the modulus of $f$ is greater than 1 in most of the annulus. Throughout we use the following function in order to simplify notation:

$$
\delta(r)=\frac{1}{\sqrt{\log r}} .
$$

Theorem 2.4. Let $f$ be a transcendental entire function, let $k>1$ and let $r$ be such that $r \geq R_{0}$, where $R_{0}=R_{0}(f)$ is the constant in Lemma 2.1, and also such that

$$
M(r)>r^{9} \text { and } \delta(r)<\min \{1 /(2 \pi),(k-1) / 4\} .
$$

$$
m(s)>1, \quad \text { for } s \in\left(r^{1+\delta(r)}, r^{k-\delta(r)}\right),
$$

then the following properties hold:

(a) We have

$$
\log m(s) \geq(1-2 \pi \delta(r)) \log M(s)>0, \quad \text { for } s \in\left[r^{1+2 \delta(r)}, r^{k-2 \delta(r)}\right] .
$$


(b) Let $R=M(r)$ and define $K=K(r)$ by

$$
R^{K}=\left(M\left(r^{k-2 \delta(r)}\right)\right)^{1-2 \pi \delta(r)} .
$$

Then $R>r^{9}$ and

$$
f\left(A\left(r, r^{k-2 \delta(r)}\right)\right) \supset A\left(R, R^{K}\right) \text { and } K \geq k(1-9 \delta(r)) .
$$

(c) If, in addition,

$$
\delta(r)<\min \{1 /(6 \pi),(k-1) /(6 \pi+1)\},
$$

then

$$
f\left(A\left(r^{1+6 \pi \delta(r)}, r^{k(1-6 \pi \delta(r))}\right)\right) \subset A\left(R^{1+6 \pi \delta(R)}, R^{K(1-6 \pi \delta(R))}\right) .
$$

Proof. The proof of part (a) is based on an application of Harnack's inequality to the harmonic function $u(t)=\log \left|f\left(e^{t}\right)\right|$, which is possible by (2.11). Since this part of the proof is identical to that of [14, Lemma 5 part (a)], we omit the details.

To prove part (b) first note that, by hypothesis,

$$
R=M(r)>r^{9}, \text { so } \frac{\delta(r)}{\delta(R)}=\sqrt{\frac{\log R}{\log r}}>3 .
$$

Evidently

$$
|f(z)| \leq M(r)=R, \text { for }|z|=r,
$$

and, by part (a) and the definition of $K$,

$$
m\left(r^{k-2 \delta(r)}\right) \geq M\left(r^{k-2 \delta(r)}\right)^{1-2 \pi \delta(r)}=R^{K}
$$

Also, by Lemma 2.1 part (b),

$$
\begin{aligned}
R^{K} & =M\left(r^{k-2 \delta(r)}\right)^{1-2 \pi \delta(r)} \\
& \geq M(r)^{(k-2 \delta(r))(1-2 \pi \delta(r))} \\
& \geq M(r)^{k(1-9 \delta(r))} \\
& =R^{k(1-9 \delta(r))} .
\end{aligned}
$$

Taken together, (2.16), (2.17) and (2.18) imply (2.12).

To prove part (c), we note that $6 \pi \delta(r)<1$ and $1+6 \pi \delta(r) \in(1+\delta(r), k-\delta(r))$, by (2.13). Thus, by part (a), Lemma 2.1 part (b), and (2.15),

$$
\begin{aligned}
m\left(r^{1+6 \pi \delta(r)}\right) & \geq M\left(r^{1+6 \pi \delta(r)}\right)^{1-2 \pi \delta(r)} \\
& \geq M(r)^{(1+6 \pi \delta(r))(1-2 \pi \delta(r))} \\
& \geq M(r)^{1+2 \pi \delta(r)} \\
& \geq M(r)^{1+6 \pi \delta(R)} \\
& =R^{1+6 \pi \delta(R)}
\end{aligned}
$$


Also, by Lemma 2.1 part (b), (2.13), (2.15), and the facts that $k(1-2 \delta(r))<$ $k-2 \delta(r)$ and $6 \pi \delta(r)<1$,

$$
\begin{aligned}
M\left(r^{k(1-6 \pi \delta(r))}\right) & \leq M\left(r^{k(1-2 \delta(r))}\right)^{\frac{1-6 \pi \delta(r)}{1-2 \delta(r)}} \\
& <R^{\frac{K}{1-2 \pi \delta(r)} \frac{1-6 \pi \delta(r)}{1-2 \delta(r)}} \\
& \leq R^{K(1-(6 \pi-2-2 \pi) \delta(r))} \\
& \leq R^{K(1-6 \pi \delta(R))} .
\end{aligned}
$$

Taken together, the estimates (2.19) and (2.20) imply (2.14), since $f$ has no zeros in the annulus $A\left(r^{1+6 \pi \delta(r)}, r^{k(1-6 \pi \delta(r))}\right)$.

\section{Proof of Theorem 1.1: Multiply connected Fatou components}

In this section we prove a version of Theorem 1.1 in which it is assumed that $f$ has a multiply connected Fatou component, $U$ say. In this case the dynamical behaviour of $f$ is very distinctive: the image domains $U_{n}=f^{n}(U), n \in \mathbb{N}$, are Fatou components (see [10, Corollary 1]) that form a sequence of eventually nested 'ring-like' domains, as described in the following result of Baker [1, Theorem 3.1].

Lemma 3.1. Let $f$ be a transcendental entire function with a multiply connected Fatou component $U$. Then the Fatou components $U_{n}=f^{n}(U), n \in \mathbb{N}$, have the following properties:

(a) each $U_{n}$ is bounded and multiply connected;

(b) there exists $N \in \mathbb{N}$ such that $U_{n}$ and 0 lie in a bounded complementary component of $U_{n+1}$, for $n \geq N$;

(c) $\operatorname{dist}\left(U_{n}, 0\right) \rightarrow \infty$ as $n \rightarrow \infty$.

Such multiply connected Fatou components $U_{n}$ are also known to contain large annuli centred at 0 , as shown by the following result from [4, Theorem 1.2] which strengthens an earlier result of Zheng [18.

Lemma 3.2. Let $f$ be a transcendental entire function with a multiply connected Fatou component $U$. For each $z_{0} \in U$ and each open set $D \subset U$ containing $z_{0}$, there exists $\alpha>0$ such that, for sufficiently large $n \in \mathbb{N}$,

$$
U_{n} \supset f^{n}(D) \supset A\left(\left|f^{n}\left(z_{0}\right)\right|^{1-\alpha},\left|f^{n}\left(z_{0}\right)\right|^{1+\alpha}\right) .
$$

We use Lemma 3.2 to prove a general result about the existence of sequences of absorbing annuli in such multiply connected Fatou components, which in a sense strengthens the results in [4]. Recall that $\delta(r)=1 / \sqrt{\log r}$.

Lemma 3.3. Let $f$ be a transcendental entire function with a multiply connected Fatou component. Then there exist sequences $\left(r_{n}\right)$ and $\left(k_{n}\right)$, with $r_{n}>0$ and $k_{n}>1$, for $n \geq 0$, such that the annuli

$$
A_{n}=A\left(r_{n}, r_{n}^{k_{n}}\right), \quad n \geq 0,
$$

and

$$
A_{n}^{\prime}=A\left(r_{n}^{1+6 \pi \delta_{n}}, r_{n}^{k_{n}\left(1-6 \pi \delta_{n}\right)}\right), \text { where } \delta_{n}=\delta\left(r_{n}\right), n \geq 0
$$


have the following properties:

(a) For $n \geq 0$,

$$
f\left(A_{n}\right) \supset A_{n+1}
$$

and

$$
f\left(A_{n}^{\prime}\right) \subset A_{n+1}^{\prime} .
$$

(b) The annuli $A_{n}^{\prime}, n \geq 0$, lie in distinct multiply connected Fatou components $U_{n}$ of $f$ and $f\left(U_{n}\right)=U_{n+1}$, for $n \geq 0$.

(c) For $n \geq 0$,

$$
\begin{gathered}
r_{n+1}=M\left(r_{n}\right)>r_{n}^{16}, \\
r_{n+1}^{k_{n+1}}=M\left(r_{n}^{k_{n}-2 \delta_{n}}\right)^{1-2 \pi \delta_{n}},
\end{gathered}
$$

and

$$
1+20 \delta_{0} \leq k_{0}<\frac{\log r_{1}}{\log r_{0}}, \quad k_{n+1} \geq k_{n}\left(1-9 \delta_{n}\right) \geq 1+20 \delta_{n+1} \text { and } r_{n}^{k_{n}}<r_{n+1} .
$$

Proof. By Lemma 3.2, there exist a multiply connected Fatou component $U$ of $f$, a point $z_{0} \in U$ and a constant $\alpha>0$ such that the Fatou components $U_{n}=f^{n}(U)$, $n \geq 0$, satisfy

$$
U_{n} \supset A\left(\left|f^{n}\left(z_{0}\right)\right|^{1-\alpha},\left|f^{n}\left(z_{0}\right)\right|^{1+\alpha}\right), \text { for } n \geq 0 .
$$

Therefore, by replacing $U$ by $U_{n}$ for a suitably large $n$ and relabeling, we can take $r_{0}>0$ such that

$$
\begin{gathered}
M(r)>r^{16}, \text { for } r \geq r_{0}, \\
U \supset A\left(r_{0}, r_{0}^{k_{0}}\right),
\end{gathered}
$$

where

$$
1+20 \delta_{0} \leq k_{0}<\frac{\log M\left(r_{0}\right)}{\log r_{0}} \text { and } \delta_{0}=\delta\left(r_{0}\right) \leq \frac{1}{80},
$$

and also, by Lemma 3.1

$$
\left|f^{n}(z)\right|>1, \text { for } z \in A\left(r_{0}, r_{0}^{k_{0}}\right), n \geq 0 .
$$

Suppose now that for some $m \geq 0$ the sequences $r_{0}, \ldots, r_{m}$ and $k_{0}, \ldots, k_{m}$ have been chosen so that they satisfy (3.3), (3.4), (3.5), (3.6) and (3.7), for $n=$ $0, \ldots, m-1$. We show that $r_{m+1}$ and $k_{m+1}$ can be chosen so that these properties also hold for $n=m$.

By (3.7), (3.8) and the fact that $6 \pi+1<20$, we can apply Theorem 2.4 with $r=r_{m}$ and $k=k_{m}$ to deduce that

$$
f\left(A_{m}\right) \supset A\left(R, R^{K}\right),
$$

where

$$
R=M\left(r_{m}\right)>r_{m}^{16}, \quad R^{K}=M\left(r_{m}^{k_{m}-2 \delta_{m}}\right)^{1-2 \pi \delta_{m}} \text { and } K \geq k_{m}\left(1-9 \delta_{m}\right),
$$

and

$$
f\left(A\left(r_{m}^{1+6 \pi \delta_{m}}, r_{m}^{k_{m}\left(1-6 \pi \delta_{m}\right)}\right)\right) \subset A\left(R^{1+6 \pi \delta(R)}, R^{K(1-6 \pi \delta(R))}\right) .
$$

We now take

$$
r_{m+1}=R, \quad \delta_{m+1}=\delta(R) \text { and } k_{m+1}=K .
$$


Then, by (3.12),

by (3.13),

$$
f\left(A_{m}\right) \supset A\left(r_{m+1}, r_{m+1}^{k_{m+1}}\right)
$$

$$
k_{m+1} \geq k_{m}\left(1-9 \delta_{m}\right)
$$

and, by (3.8),

$$
\frac{\delta_{j}}{\delta_{j+1}}=\frac{\sqrt{\log r_{j+1}}}{\sqrt{\log r_{j}}}=\sqrt{\frac{\log M\left(r_{j}\right)}{\log r_{j}}} \geq 4, \text { for } j=0, \ldots, m .
$$

Therefore, by (3.10),

$$
k_{m+1} \geq\left(1+20 \delta_{0}\right) \prod_{j=0}^{m}\left(1-9 \delta_{j}\right) \geq\left(1+20 \delta_{0}\right)\left(1-12 \delta_{0}\right) \geq 1+5 \delta_{0} \geq 1+20 \delta_{m+1} .
$$

Finally, the inequality $r_{m}^{k_{m}}<M\left(r_{m}\right)=r_{m+1}$ holds in the case $m=0$, by (3.10), and we deduce it in the case $m \geq 1$ from (3.6) and (3.7) with $n=m-1$, since these imply that

$$
r_{m}^{k_{m}} \leq M\left(r_{m-1}^{k_{m-1}}\right)<M\left(r_{m}\right)=r_{m+1} .
$$

We have now checked that (3.3), (3.4), (3.5), (3.6) and (3.7) all hold with $n=m$, and by induction this completes the proofs of parts (a) and (c).

To prove part (b), note that (3.1), (3.2), (3.3) and (3.9) imply that for $n \geq 0$ we have $A_{n}^{\prime} \subset A_{n} \subset f^{n}(U)$, and the sets $U_{n}=f^{n}(U), n \geq 0$, form disjoint Fatou components by Lemma 3.1 .

We now use Lemma 3.3 to prove the stronger version of Theorem 1.1 that holds when $f$ has a multiply connected Fatou component. Here, for a closed annulus $B=\bar{A}(r, s), 0<r<s$, we denote by $\partial_{\text {inn }} B$ and $\partial_{\text {out }} B$ the inner and outer boundary components of $B$, respectively.

Theorem 3.4. Let $f$ be a transcendental entire function with a multiply connected Fatou component. Then there exist $R=R(f)>0$, a sequence $\left(U_{n}\right)$ of distinct multiply connected Fatou components of $f$ such that $f\left(U_{n}\right)=U_{n+1}$, for $n \geq 0$, and a sequence of closed annuli

$$
B_{n}=\bar{A}\left(t_{n}, t_{n}^{\prime}\right), \quad n \geq 0,
$$

such that

$$
\partial_{\text {inn }} B_{n} \subset U_{n} \text { and } \partial_{\text {out }} B_{n} \subset U_{n+1}, \text { for } n \geq 0,
$$

so each $B_{n}$ meets $J(f)$,

$$
\begin{gathered}
{\left[t_{n+1}, t_{n+1}^{\prime}\right] \subset\left[M\left(t_{n}\right), M\left(t_{n}^{\prime}\right)\right], \text { for } n \geq 0,} \\
B_{n} \subset A_{n}(R), \text { for } n \geq 0, \\
f\left(B_{n}\right) \supset B_{n+1}, \text { for } n \geq 0,
\end{gathered}
$$

and there is a subsequence $\left(B_{n_{j}}\right)$ such that

$$
f\left(B_{n_{j}}\right) \supset B_{n}, \text { for } j \in \mathbb{N}, 0 \leq n \leq n_{j} .
$$

Also, the sequence $\left(n_{j}\right)$ consists of those $n \geq 0$ such that $A_{n}(R)$ contains a zero of $f$. 
Proof. Let $\left(r_{n}\right),\left(k_{n}\right),\left(A_{n}\right),\left(A_{n}^{\prime}\right)$ and $\left(U_{n}\right)$ be the sequences given by Lemma 3.3 . in particular,

$$
A_{n}^{\prime} \subset U_{n} \text { and } f\left(A_{n}^{\prime}\right) \subset A_{n+1}^{\prime}, \text { for } n \geq 0 \text {, }
$$

by (3.4).

Now define the sequence of closed annuli $\left(B_{n}\right)$ as follows:

$$
B_{n}=\bar{A}\left(t_{n}, t_{n}^{\prime}\right), \text { for } n \geq 0,
$$

where

$$
t_{n}=r_{n}^{k_{n}\left(1-6 \pi \delta_{n}\right)} \text { and } t_{n}^{\prime}=r_{n+1}^{1+6 \pi \delta_{n+1}} \text {, for } n \geq 0 ;
$$

that is, $B_{n}$ is the annulus lying between $A_{n}^{\prime}$ and $A_{n+1}^{\prime}$. Since $A_{n}^{\prime}$ and $A_{n+1}^{\prime}$ lie in distinct Fatou components, it follows that $B_{n} \cap J(f) \neq \emptyset$, for $n \geq 0$.

Also, by (3.19), we have

$$
f\left(\partial_{\text {inn }} B_{n}\right) \subset \overline{A_{n+1}^{\prime}} \text { and } f\left(\partial_{\text {out }} B_{n}\right) \subset \overline{A_{n+2}^{\prime}} \text {, for } n \geq 0,
$$

SO

$$
f\left(B_{n}\right) \supset B_{n+1}, \text { for } n \geq 0,
$$

which proves (3.17).

We also have in this case, by (3.2) and (3.21), that

$$
t_{n}^{\prime}<M\left(t_{n}\right) \leq r_{n+1}^{k_{n+1}\left(1-6 \pi \delta_{n+1}\right)}=t_{n+1}, \text { for } n \geq 0,
$$

and, by (3.7), (3.20) and (3.21), that

$$
t_{n+1}<r_{n+1}^{k_{n+1}}<M\left(r_{n+1}\right)=r_{n+2}<t_{n+1}^{\prime}<M\left(t_{n}^{\prime}\right), \text { for } n \geq 0 .
$$

Together, (3.22) and (3.23) prove (3.15)

Now (3.22) implies that there exists $R=R(f)>0$ such that

$$
t_{0}^{\prime}<R<t_{1}<t_{1}^{\prime}<M(R),
$$

so $B_{0} \subset A_{0}(R)$ and $B_{1} \subset A_{1}(R)$, and we deduce by (3.15) that

$$
M^{n-1}(R)<t_{n}<t_{n}^{\prime}<M^{n}(R) \text {, so } B_{n} \subset A_{n}(R) \text {, for } n \in \mathbb{N} \text {, }
$$

which proves (3.16).

To prove the final statement, let

$$
B_{n}^{\prime}=A_{n}^{\prime} \cup B_{n} \cup A_{n+1}^{\prime}, \quad n \geq 0,
$$

and let $F_{n}$ denote the component of $\mathbb{C} \backslash B_{n}^{\prime}$ that contains 0 . Then, by (3.19),

$$
\partial f\left(B_{n}^{\prime}\right) \subset f\left(\partial B_{n}^{\prime}\right) \subset B_{n+1}^{\prime}, \text { for } n \geq 0,
$$

and hence, for each $n \geq 0$, we have exactly one of the following possibilities:

$$
f\left(B_{n}^{\prime}\right) \subset B_{n+1}^{\prime}
$$

or

$$
f\left(B_{n}^{\prime}\right) \supset F_{n+1}, \text { so } f\left(B_{n}\right) \supset F_{n+1} .
$$

If (3.24) holds for all $n \geq N$, say, then each $B_{n}^{\prime}, n \geq N$, is contained in the Fatou set of $f$, by Montel's theorem, and this contradicts the fact that each $B_{n}$ and hence each $B_{n}^{\prime}$ meets $J(f)$. Thus there is a strictly increasing sequence $n_{j} \geq 0$ such that (3.25) holds for all $n=n_{j}, j \in \mathbb{N}$, which gives (3.18). By replacing $R$ by $M^{n}(R)$ for some $n$, we can assume that $n_{0}=0$. By (3.24) and (3.25), this sequence $\left(n_{j}\right)$ consists of those $n \geq 0$ such that $B_{n}$ contains a zero of $f$. Hence, by (3.16) 
and (3.19), this sequence consists of those $n \geq 0$ such that $A_{n}(R)$ contains a zero of $f$.

\section{Proof of Theorem 1.1; No multiply connected Fatou components}

The proof of Theorem 1.1 in the case of no multiply connected Fatou components is somewhat more complicated and is based on the following lemma. Recall again that $\delta(r)=1 / \sqrt{\log r}$.

Lemma 4.1. Let $f$ be a transcendental entire function with no multiply connected Fatou components. There exists $R_{3}=R_{3}(f)>0$ such that for all $r_{0} \geq R_{3}$ and $k_{0}=1+20 \delta\left(r_{0}\right)$ there exist finite sequences $r_{n}>0$ and $k_{n}>1, n=0, \ldots, N$, where $N \geq 0$, such that the annuli

$$
A_{n}=A\left(r_{n}, r_{n}^{k_{n}}\right), \quad n=0, \ldots, N,
$$

and

$$
A_{n}^{\prime}=A\left(r_{n}^{1+6 \pi \delta_{n}}, r_{n}^{k_{n}\left(1-6 \pi \delta_{n}\right)}\right), \text { where } \delta_{n}=\delta\left(r_{n}\right), n=0, \ldots, N,
$$

have the following properties:

(a) For $n=0, \ldots, N-1$,

$$
f\left(A_{n}\right) \supset A_{n+1}
$$

and

(b) For any $S, S^{\prime}, T, T^{\prime}$ that satisfy

$$
2<S<S^{\prime}, \quad T<T^{\prime} \leq M\left(\frac{1}{3} r_{N}^{1+\delta_{N}}\right) \text { and } S^{\prime} \leq \frac{1}{2} T,
$$

we have

$$
f\left(A_{N}\right) \text { contains } A\left(S, S^{\prime}\right) \text { or } A\left(T, T^{\prime}\right) .
$$

(c) For $n=0, \ldots, N-1$,

$$
\begin{gathered}
r_{n+1}=M\left(r_{n}\right)>r_{n}^{16}, \\
r_{n+1}^{k_{n+1}}=M\left(r_{n}^{k_{n}-2 \delta_{n}}\right)^{1-2 \pi \delta_{n}}, \\
k_{n+1} \geq k_{n}\left(1-9 \delta_{n}\right) \geq 1+5 \delta_{0} \geq 1+20 \delta_{n+1},
\end{gathered}
$$

and

$$
M\left(r_{n}^{k_{n}}\right)>r_{n+1}^{k_{n+1}}>r_{n+1}=M\left(r_{n}\right)>\left(r_{n}^{k_{n}}\right)^{2} .
$$

Also,

Proof. We construct the annuli $A_{n}$ by using Theorem 2.4 in a way that is similar to the proof of Lemma 3.3 but rather more complicated.

We take $R_{3} \geq \max \left\{R_{0}^{2}, R_{2}\right\}$, where $R_{0}=R_{0}(f)$ is the constant in Lemma 2.1 and $R_{2}=R_{2}(f)$ is the constant in Corollary 2.3 , and also such that

$$
M(r)>r^{16} \text { and } \sqrt{\log r} \geq 80 \text {, for } r \geq R_{3} .
$$

Given $r_{0} \geq R_{3}$, we define the annulus $A_{0}=A\left(r_{0}, r_{0}^{k_{0}}\right)$, where $k_{0}=1+20 \delta_{0}$ with $\delta_{0}=\delta\left(r_{0}\right)$. Suppose that, for some $m \geq 0$, the sequences $r_{0}, \ldots, r_{m}$ and $k_{0}, \ldots, k_{m}$ have been chosen so that they satisfy (4.1), (4.2), (4.4), (4.5), (4.6) and (4.7), for $n=0, \ldots, m-1$. 
We now show that if the condition

$$
m(s)>1, \quad \text { for all } s \in\left(r_{m}^{1+\delta_{m}}, r_{m}^{k_{m}-\delta_{m}}\right),
$$

holds, then $r_{m+1}$ and $k_{m+1}$ can be chosen so that these properties also hold with $n=m$.

By (4.6), (4.9) and (4.10), we can apply Theorem 2.4 with $r=r_{m}$ and $k=k_{m}$ to deduce that

$$
f\left(A_{m}\right)=f\left(A\left(r_{m}, r_{m}^{k_{m}}\right)\right) \supset A\left(R, R^{K}\right)
$$

where

$$
R=M\left(r_{m}\right)>r_{m}^{16}, \quad R^{K}=M\left(r_{m}^{k_{m}-2 \delta_{m}}\right)^{1-2 \pi \delta_{m}} \text { and } K \geq k_{m}\left(1-9 \delta_{m}\right),
$$

and

$$
f\left(A\left(r_{m}^{1+6 \pi \delta_{m}}, r_{m}^{k_{m}\left(1-6 \pi \delta_{m}\right)}\right)\right) \subset A\left(R^{1+6 \pi \delta(R)}, R^{K(1-6 \pi \delta(R))}\right) .
$$

So if we take

$$
r_{m+1}=R, \quad \delta_{m+1}=\delta(R) \text { and } k_{m+1}=K,
$$

then (4.11), (4.12) and (4.13) imply that the properties (4.1), (4.2), (4.4) and (4.5) all hold with $n=m$.

To deduce (4.6) in the case $n=m$ first note that, by (4.9),

$$
\delta_{0} \leq \frac{1}{80} \text { and } \frac{\delta_{j}}{\delta_{j+1}}=\frac{\sqrt{\log r_{j+1}}}{\sqrt{\log r_{j}}}=\sqrt{\frac{\log M\left(r_{j}\right)}{\log r_{j}}} \geq 4, \text { for } j=0, \ldots, m .
$$

Thus, by (4.6) with $n=0, \ldots, m-1$, (4.12), and the fact that $k_{m+1}=K$,

$$
k_{m+1} \geq k_{0} \prod_{j=0}^{m}\left(1-9 \delta_{j}\right) \geq\left(1+20 \delta_{0}\right)\left(1-12 \delta_{0}\right) \geq 1+5 \delta_{0} \geq 1+20 \delta_{m+1},
$$

as required.

The first two inequalities in (4.7) with $n=m$ follow immediately from (4.5) and (4.6). Also, the inequality $\left(r_{m}^{k_{m}}\right)^{2}<M\left(r_{m}\right)=r_{m+1}$ holds in the case $m=0$, by (4.9), and we can deduce this inequality in the case $m \geq 1$ from (4.5), (4.7) with $n=m-1$, and Lemma 2.1 part (b) (applied with $r=r_{m-1}^{k_{m-1}}$ and $k=2$ ) as follows:

$$
\begin{aligned}
\left(r_{m}^{k_{m}}\right)^{2} & =\left(M\left(r_{m-1}^{k_{m-1}-2 \delta_{m-1}}\right)^{1-2 \pi \delta_{m-1}}\right)^{2} \leq\left(M\left(r_{m-1}^{k_{m-1}}\right)\right)^{2} \\
& \leq M\left(\left(r_{m-1}^{k_{m-1}}\right)^{2}\right)<M\left(r_{m}\right)=r_{m+1} .
\end{aligned}
$$

Hence (4.1), (4.2), (4.4), (4.5), (4.6) and (4.7) all hold for $n=m$.

We now observe that the condition in (4.10) cannot hold for all $m \geq 0$. For if this were the case, then we could construct a sequence of annuli $\left(A_{n}\right)_{0}^{\infty}$ satisfying (4.1), (4.2), (4.4), (4.5) and (4.6), and property (4.2) would then imply that $f$ has multiply connected Fatou components, contrary to our hypothesis.

Therefore for some $N \geq 0$ the condition in (4.10) holds for $m=0, \ldots, N-1$, and the annuli $A\left(r_{n}, r_{n}^{k_{n}}\right), n=0, \ldots, N-1$, exist satisfying properties (a) and (c), including (4.8) by (4.14), but (4.10) fails for $m=N$; that is,

$$
\text { there exists } s \in\left(r_{N}^{1+\delta_{N}}, r_{N}^{k_{N}-\delta_{N}}\right) \text { such that } m(s) \leq 1 \text {. }
$$

Then

$$
s \in\left(\frac{2}{3} s, \frac{4}{3} s\right) \subset\left(\frac{1}{3} s, \frac{8}{3} s\right) \subset\left(\frac{1}{3} r_{N}^{1+\delta_{N}}, \frac{8}{3} r_{N}^{k_{N}-\delta_{N}}\right) \subset\left(r_{N}, r_{N}^{k_{N}}\right),
$$


since $r_{N}^{\delta_{N}}=\exp \left(\sqrt{\log r_{N}}\right) \geq 3$. Therefore, since $m(s) \leq 1$, we can apply Corollary 2.3 with $r=\frac{1}{3} s$ to deduce that if $S, S^{\prime}, T, T^{\prime}$ satisfy

$$
2<S<S^{\prime}, \quad T<T^{\prime} \leq M\left(\frac{1}{3} r_{N}^{1+\delta_{N}}\right) \text { and } S^{\prime} \leq \frac{1}{2} T,
$$

then

so, by (4.16),

$$
f\left(A\left(\frac{1}{3} s, \frac{8}{3} s\right)\right) \text { contains } A\left(S, S^{\prime}\right) \text { or } A\left(T, T^{\prime}\right)
$$

$$
f\left(A_{N}\right) \text { contains } A\left(S, S^{\prime}\right) \text { or } A\left(T, T^{\prime}\right)
$$

as required.

We now use Lemma4.1 to prove Theorem 1.1 in the case when $f$ has no multiply connected Fatou components.

Theorem 4.2. Let $f$ be a transcendental entire function with no multiply connected Fatou components. There exists $R=R(f)>0$ and a sequence of closed annuli

$$
B_{n}=\bar{A}\left(r_{n}, r_{n}^{k_{n}}\right), \quad n \geq 0,
$$

each of which meets $J(f)$, such that

$$
B_{n} \subset A_{n}(R), \text { for } n \geq 0,
$$

and

$$
f\left(B_{n}\right) \supset B_{n+1}, \text { for } n \geq 0,
$$

and there is a subsequence $B_{n_{j}}$ such that, for $j \in \mathbb{N}$,

$$
f\left(B_{n_{j}}\right) \supset B_{n} \text {, for } 0 \leq n \leq n_{j} \text {, with at most one exception. }
$$

Proof. We begin by taking $R_{4} \geq \max \left\{R_{0}, R_{2}, R_{3}\right\}$, where $R_{0}$ is the constant in Lemma 2.1, $R_{2}$ is the constant in Corollary 2.3, and $R_{3}$ is the constant in Lemma 4.1, and also such that

$$
M(r)>r^{10000} \text { and } \sqrt{\log r} \geq 80, \text { for } r \geq R_{4} .
$$

In the proof we construct annuli $B_{n}$ of the form given in (4.17) which satisfy the conclusions of the theorem and also satisfy

$$
M\left(r_{n}^{k_{n}}\right)>r_{n+1}^{k_{n+1}}>r_{n+1} \geq M\left(r_{n}\right)>\left(r_{n}^{k_{n}}\right)^{2}, \text { for } n \geq 0,
$$

a sequence of estimates that is closely related to (4.7).

Take $r_{0} \geq R_{4}$. Then, by Lemma 4.1, there exists $N(1) \geq 0$ and annuli

$$
A_{n}=A\left(r_{n}, r_{n}^{k_{n}}\right), \quad n=0, \ldots, N(1),
$$

with the properties given in that lemma.

Putting

$$
B_{n}=\bar{A}_{n}, \text { for } n=0, \ldots, N(1),
$$

we deduce, by (4.1), that

$$
f\left(B_{n}\right) \supset B_{n+1}, \text { for } n=0, \ldots, N(1)-1,
$$

and, by (4.7), that

$$
M\left(r_{n}^{k_{n}}\right)>r_{n+1}^{k_{n+1}}>r_{n+1}=M\left(r_{n}\right)>\left(r_{n}^{k_{n}}\right)^{2}, \text { for } n=0, \ldots, N(1)-1 .
$$

Also, by Lemma 4.1 part (b), for any $S, S^{\prime}, T, T^{\prime}$ that satisfy

$$
2<S<S^{\prime}, \quad T<T^{\prime} \leq M\left(\frac{1}{3} r_{N(1)}^{1+\delta_{N(1)}}\right) \text { and } S^{\prime} \leq \frac{1}{2} T,
$$


we have

$$
f\left(B_{N(1)}\right) \text { contains } \bar{A}\left(S, S^{\prime}\right) \text { or } \bar{A}\left(T, T^{\prime}\right) \text {. }
$$

We apply the covering property (4.26) in two situations. First we take $S, S^{\prime}$, $T, T^{\prime}$ as follows:

$$
S=M\left(r_{N(1)}\right) \text { and } S^{\prime}=S^{1+20 \delta(S)}
$$

and

$$
T=S^{1+40 \delta(S)} \text { and } T^{\prime}=T^{1+20 \delta(T)} .
$$

This choice is possible since, by (4.21),

$$
\frac{T}{S^{\prime}}=S^{20 \delta(S)}=\exp (20 \sqrt{\log S}) \geq 2,
$$

and, by Lemma 2.1 part (b), (4.27), (4.28) and the fact that $\sqrt{\log r_{N(1)}} \geq 5 \log 3$,

$$
\begin{aligned}
M\left(\frac{1}{3} r_{N(1)}^{1+\delta_{N(1)}}\right) & =M\left(r_{N(1)}^{1+\delta_{N(1)}-\log 3 / \log r_{N(1)}}\right) \geq M\left(r_{N(1)}^{1+\frac{4}{5} \delta_{N(1)}}\right) \\
& \geq M\left(r_{N(1)}\right)^{1+\frac{4}{5} \delta_{N(1)}}=S^{1+\frac{4}{5} \delta_{N(1)}} \\
& \geq S^{(1+40 \delta(S))(1+20 \delta(T))}=T^{\prime},
\end{aligned}
$$

since (4.21) implies that $\delta(S) \leq \delta_{N(1)} / 100 \leq 1 / 80$.

Thus (4.26) holds for these choices of $S, S^{\prime}, T, T^{\prime}$. Hence we can define the pair $\left(r_{N(1)+1}, \delta_{N(1)+1}\right)$ to be either $(S, \delta(S))$ or $(T, \delta(T))$, and then define

$$
k_{N(1)+1}=1+20 \delta_{N(1)+1}
$$

and

$$
B_{N(1)+1}=\bar{A}\left(r_{N(1)+1}, r_{N(1)+1}^{k_{N(1)+1}}\right),
$$

to ensure that (4.19) holds for $n=N(1)$. Then (4.22) also holds for this value of $n$; that is,

$$
M\left(r_{N(1)}^{k_{N(1)}}\right)>r_{N(1)+1}^{k_{N(1)+1}}>r_{N(1)+1} \geq M\left(r_{N(1)}\right)>\left(r_{N(1)}^{k_{N(1)}}\right)^{2} .
$$

Indeed, by the definition of $r_{N(1)+1}$, the definition of $S$ and (4.8),

$$
r_{N(1)+1} \geq S=M\left(r_{N(1)}\right)>\left(r_{N(1)}^{k_{N(1)}}\right)^{2},
$$

and, by Lemma 2.1 part (b) and the definition of $k_{N(1)+1}$,

$$
\begin{aligned}
& M\left(r_{N(1)}^{k_{N(1)}}\right) \geq M\left(r_{N(1)}\right)^{k_{N(1)}} \\
& =S^{k_{N(1)}} \geq S^{1+20 \delta_{N(1)}} \\
& \geq S^{(1+40 \delta(S))(1+20 \delta(S))} \geq T^{1+20 \delta_{N(1)+1}} \\
& =T^{k_{N(1)+1}} \geq r_{N(1)+1}^{k_{N(1)+1}},
\end{aligned}
$$

since $\delta_{N(1)+1}=\delta\left(r_{N(1)+1}\right) \leq \delta(S) \leq \delta_{N(1)} / 100 \leq 1 / 80$.

We also apply the covering property (4.26) when

$$
A\left(S, S^{\prime}\right)=A\left(r_{p}, r_{p}^{k_{p}}\right) \text { and } A\left(T, T^{\prime}\right)=A\left(r_{q}, r_{q}^{k_{q}}\right),
$$

where $0 \leq p<q \leq N(1)$, which is possible since

$$
r_{n}^{k_{n}}<r_{n+1}^{1 / 2}, \text { so } r_{n+1} \geq 2 r_{n}^{k_{n}}, \text { for } n=0, \ldots, N(1)-1,
$$


by (4.24), and

$$
r_{N(1)}^{k_{N(1)}} \leq M\left(\frac{1}{3} r_{N(1)}^{1+\delta_{N}(1)}\right)
$$

by (4.30), for example. It follows that

$$
f\left(B_{N(1)}\right) \supset B_{n}, \text { for } n=0, \ldots, N(1) \text {, with at most one exception. }
$$

Now, since

$$
B_{N(1)+1}=\bar{A}\left(r_{N(1)+1}, r_{N(1)+1}^{k_{N(1)+1}}\right), \text { where } k_{N(1)+1}=1+20 \delta_{N(1)+1},
$$

we can apply Lemma 4.1 with $r_{0}$ replaced by $r_{N(1)+1}$ to give $N(2) \in \mathbb{N}$ with $N(2)>N(1)$ and closed annuli $B_{n}, n=N(1)+1, \ldots, N(2)$, which satisfy (4.22) and (4.19) for $n=N(1)+1, \ldots, N(2)$, and also

$$
f\left(B_{N(2)}\right) \supset B_{n}, \text { for } n=0, \ldots, N(2) \text {, with at most one exception. }
$$

Repeating this application of Lemma 4.1 infinitely often, and introducing the subsequence $n_{j}=N(j), j \in \mathbb{N}$, we obtain a sequence $\left(B_{n}\right)$ that satisfies (4.19), (4.20) and (4.22). Also, since $f$ has no multiply connected Fatou components, all the components of $J(f)$ are unbounded (see, for example, [11, Theorem 1]), and so the closed annuli $B_{n}$ must all meet $J(f)$ by (4.19).

Finally, (4.22) implies that there exists $R=R(f)>0$ such that (4.18) holds. Indeed, by (4.22) we can choose $R>0$ such that

$$
r_{0}^{k_{0}}<R<r_{1}<r_{1}^{k_{1}}<M(R),
$$

so $B_{0} \subset A_{0}(R)$ and $B_{1} \subset A_{1}(R)$, and, by (4.22) again,

$$
M^{n-1}(R)<r_{n}<r_{n}^{k_{n}}<M^{n}(R) \text {, so } B_{n} \subset A_{n}(R) \text {, for } n \in \mathbb{N} \text {, }
$$

which proves (4.18).

\section{Proof of Theorem 1.2}

We deduce Theorem 1.2 from Theorem 1.1 by using the following simple topological lemma, proved in [14, Lemma 1].

Lemma 5.1. Let $E_{n}, n \geq 0$, be a sequence of compact sets in $\mathbb{C}$ and $f: \mathbb{C} \rightarrow \mathbb{C}$ be a continuous function such that

$$
f\left(E_{n}\right) \supset E_{n+1}, \quad \text { for } n \geq 0 .
$$

Then there exists $\zeta$ such that $f^{n}(\zeta) \in E_{n}$, for $n \geq 0$.

If $f$ is also meromorphic and $E_{n} \cap J(f) \neq \emptyset$, for $n \geq 0$, then there exists $\zeta \in J(f)$ such that $f^{n}(\zeta) \in E_{n}$, for $n \geq 0$.

Proof of Theorem 1.2. By Theorem 1.1, there exists $R=R(f)>0$ and a sequence of closed annuli

$$
B_{n}=\bar{A}\left(r_{n}, r_{n}^{\prime}\right), \quad n \geq 0,
$$

each of which meets $J(f)$, such that

$$
\begin{gathered}
B_{n} \subset A_{n}(R), \text { for } n \geq 0, \\
f\left(B_{n}\right) \supset B_{n+1}, \text { for } n \geq 0,
\end{gathered}
$$

and there is a subsequence $B_{n_{j}}$ such that, for $j \in \mathbb{N}$,

$$
f\left(B_{n_{j}}\right) \supset B_{n} \text {, for } 0 \leq n \leq n_{j} \text {, with at most one exception. }
$$


To prove Theorem 1.2 we take this value of $R$, this sequence $\left(n_{j}\right)$ of non-negative integers and, for $j \in \mathbb{N}$, let $I_{j}$ be the singleton set consisting of the exceptional integer in $\left\{0, \ldots, n_{j}\right\}$ if this occurs in (5.4), and the empty set otherwise.

If $s_{0} s_{1} \ldots$ is any sequence of non-negative integers satisfying the hypotheses of Theorem 1.2, then it follows from (5.3) and (5.4) that the compact sets

$$
E_{n}=B_{s_{n}} \subset A_{s_{n}}(R), \text { for } n \geq 0,
$$

satisfy (5.1). Thus, by Lemma 5.1, there exists a point $\zeta \in J(f)$ with itinerary $s_{0} s_{1} \ldots$ This proves the first part of Theorem [1.2, and the last part follows immediately from the last part of Theorem 1.1

We now deduce Corollary 1.3 .

Proof of Corollary 1.3. Parts (a), (b) and (c) follow easily from Theorem 1.2, by using appropriate sequences $s_{0} s_{1} \ldots$ chosen to satisfy property (1.5) and $s_{n} \geq s$ for $n \geq 0$. The uncountable number of itineraries in parts (b) and (c) follows from the fact that if $m$ and $j$ are such that $s_{m}=n_{j}$ and $s_{m} \geq s+2$, then there are at least two possible choices for $s_{m+1}$ from the set $\{s, s+1, s+2\}$.

Part (d) is proved as follows. Let $\left(a_{n}\right)$ be any positive sequence such that $a_{n} \rightarrow \infty$ as $n \rightarrow \infty$. Without loss of generality we can assume that $\left(a_{n}\right)$ is increasing. Then we use the fact that

$$
f^{2}\left(B_{n_{j}}\right) \supset B_{n_{j}}, \text { for } j \in \mathbb{N},
$$

which follows from (5.3) and (5.4), to choose a sequence $s_{0} s_{1} \ldots$ such that $s_{n} \rightarrow \infty$ as $n \rightarrow \infty$, property (1.5) holds and, for $j \in \mathbb{N}$,

$$
N_{j}=\max \left\{m: s_{m}=n_{j}\right\} \text { satisfies } a_{N_{j}} \geq M^{n_{j+1}}(R) .
$$

Then any point $\zeta$ with itinerary $s_{0} s_{1} \ldots$ has an orbit that visits each annulus $B_{n_{j}}$ so often that

$$
\left|f^{n}(\zeta)\right| \leq M^{s_{n}}(R) \leq a_{n}, \text { for } n \geq N_{1} .
$$

Once again we can obtain uncountably many such itineraries by using the fact, which follows from (5.5), that for each $j \in \mathbb{N}$ there are infinitely many ways of choosing the integer $N_{j}$ to satisfy (5.6).

\section{Proof of Theorem 1.4}

We prove two versions of Theorem 1.4, in the first of which it is assumed that $f$ has a multiply connected Fatou component.

Theorem 6.1. Let $f$ be a transcendental entire function with a multiply connected Fatou component. Then there exists $R=R(f)>0$ with the property that whenever $\left(a_{n}\right)$ is a positive sequence such that

$$
a_{n} \geq R \text { and } a_{n+1} \leq M\left(a_{n}\right), \text { for } n \geq 0,
$$

there is a point $\zeta \in J(f)$ and a sequence $\left(n_{j}\right)$ with $n_{j} \rightarrow \infty$ as $j \rightarrow \infty$ such that

$$
\left|f^{n}(\zeta)\right| \geq a_{n}, \text { for } n \geq 0, \text { and }\left|f^{n_{j}}(\zeta)\right| \leq M^{2}\left(a_{n_{j}}\right) \text {, for } j \in \mathbb{N} \text {. }
$$

If, in addition,

$$
\text { for each } \ell \in \mathbb{N} \text { there exists } n(\ell) \in \mathbb{N} \text { such that } a_{n(\ell)+\ell}<M^{n(\ell)}(R) \text {, }
$$

then there are uncountably many itineraries with respect to $\left\{A_{n}(R)\right\}$ that correspond to points $\zeta$ satisfying (6.2). 
Remarks. 1. It is natural to ask if the expression $M^{2}\left(a_{n_{j}}\right)$ in (6.2) can be replaced by $M\left(a_{n_{j}}\right)$.

2. Note that the hypothesis (6.3) is independent of $R$ for sufficiently large $R$.

Proof of Theorem 6.1. Let $R=R(f)>0$ and $B_{n}=\bar{A}\left(t_{n}, t_{n}^{\prime}\right), n \geq 0$, be the constant and the sequence of closed annuli that were obtained in Theorem 3.4. These annuli all meet $J(f)$ and have the following properties:

$$
\begin{gathered}
{\left[t_{n+1}, t_{n+1}^{\prime}\right] \subset\left[M\left(t_{n}\right), M\left(t_{n}^{\prime}\right)\right], \text { for } n \geq 0,} \\
B_{n} \subset A_{n}(R), \text { for } n \geq 0, \\
f\left(B_{n}\right) \supset B_{n+1}, \text { for } n \geq 0,
\end{gathered}
$$

and $\left(B_{n}\right)$ has a subsequence, here called $\left(B_{N_{j}}\right)$, such that

$$
f\left(B_{N_{j}}\right) \supset B_{n}, \text { for } j \in \mathbb{N}, 0 \leq n \leq N_{j} .
$$

Suppose that $\left(a_{n}\right)$ is any sequence that satisfies (6.1). We shall apply Lemma 5.1 to a sequence of closed annuli $\left(E_{n}\right)$, all of which are chosen from the sequence $\left(B_{n}\right)$ and satisfy

$$
E_{n} \subset\left\{z:|z| \geq a_{n}\right\}, \text { for } n \geq 0 \text {. }
$$

First define

$$
E_{0}=B_{p}, \text { where } p \text { is the least integer such that } t_{p} \geq a_{0} .
$$

Then, for each $n \geq 0$, we choose $E_{n+1}$ depending on $E_{n}$ by applying the following rule.

Suppose that $E_{n}=B_{m}$ and $t_{m} \geq a_{n}$ :

(1) if $m=N_{j}$ for some $j \in \mathbb{N}$, then

$$
E_{n+1}=B_{p}, \text { where } p \text { is the least integer such that } t_{p} \geq a_{n+1} \text {; }
$$

(2) otherwise $E_{n+1}=B_{m+1}$.

Since (6.4) and (6.1) guarantee that

$$
t_{m+1} \geq M\left(t_{m}\right) \geq M\left(a_{n}\right) \geq a_{n+1},
$$

we have $p \leq m+1$ in case (1) and, in either case,

$$
E_{n+1} \subset\left\{z:|z| \geq a_{n+1}\right\} \text {. }
$$

Also, in case (1) the definition of $p$ in (6.8) gives $a_{n+1}>t_{p-1}$ (note that $p \geq 1$ because $a_{n+1} \geq R>t_{0}$ by (6.5) ). Hence, by (6.5) and (6.4) again,

$$
M^{2}\left(a_{n+1}\right)>M^{2}\left(t_{p-1}\right)>M\left(t_{p-1}^{\prime}\right)>t_{p}^{\prime},
$$

so, in this case,

$$
E_{n+1}=B_{p} \subset\left\{z:|z| \leq M^{2}\left(a_{n+1}\right)\right\} .
$$

With this choice of the annuli $E_{n}$ it follows from (6.6) and (6.7) that

$$
f\left(E_{n}\right) \supset E_{n+1}, \text { for } n \geq 0,
$$

that

$$
E_{n} \cap J(f) \neq \emptyset \text { and } E_{n} \subset\left\{z:|z| \geq a_{n}\right\} \text {, for } n \geq 0,
$$

and that $\left(E_{n}\right)$ has a subsequence, $\left(E_{n_{j}}\right)$ say, satisfying

$$
E_{n_{j}} \subset\left\{z:|z| \leq M^{2}\left(a_{n_{j}}\right)\right\} .
$$


Therefore any $\zeta$ given by Lemma 5.1, with the property that

$$
f^{n}(\zeta) \in E_{n} \cap J(f), \text { for } n \geq 0,
$$

satisfies (6.2) with this subsequence $n_{j}$.

Finally suppose that (6.3) holds. Then, for all $\ell \in \mathbb{N}$, we deduce, by (6.1), that

$$
a_{n+\ell}<M^{n}(R), \text { for } n \geq n(\ell) .
$$

We can obtain other points $\zeta$ that satisfy (6.2) with different subsequences $\left(n_{j}\right)$ and different itineraries by using the same construction as that given above but choosing $E_{n+1}$ in case (1) as follows: whenever $E_{n}=B_{m}, m=N_{j}$ and the value of $p$ specified by (6.8) satisfies $p \leq m$, we choose either the value of $p$ that is specified by (6.8) or $p=m+1$. The conditions (6.2) and (6.9) imply that the value of $p$ specified by (6.8) satisfies $p \leq m$ infinitely often (for otherwise $E_{n}=B_{n+q}$ for some $q \in \mathbb{Z}$ and all sufficiently large $n$ ). If we vary the construction in this way and consider all possible ways of choosing $E_{n+1}$ in case (1) that correspond to a point $\zeta$ satisfying (6.2), then the itineraries with respect to $\left\{A_{n}(R)\right\}$ that arise form the branches of an infinite binary tree and so are uncountable. This completes the proof of Theorem 6.1.

In our second version of Theorem 1.4 it is assumed that $f$ has no multiply connected Fatou components. Here a very similar technique to that used in the proof of Theorem 6.1 could be used to deduce the result from the annuli $B_{n}$ obtained in Theorem 4.2 but using this approach the estimate for $f^{n_{j}}(\zeta)$ would be only $\left|f^{n_{j}}(\zeta)\right| \leq M^{3}\left(a_{n_{j}}\right)$ because of the possible exceptional annulus not covered by $f\left(B_{n_{j}}\right)$ in Theorem 4.2, However, we can obtain a much stronger result by using Lemma 4.1 directly.

In this proof we once again use the notation $\delta(r)=1 / \sqrt{\log r}$.

Theorem 6.2. Let $f$ be a transcendental entire function with no multiply connected Fatou components and let $\varepsilon \in(0,1)$. Then there exists $R=R(f, \varepsilon)>0$ with the property that whenever $\left(a_{n}\right)$ is a positive sequence such that

$$
a_{n} \geq R \text { and } a_{n+1} \leq M\left(a_{n}\right), \text { for } n \geq 0,
$$

there exists a point $\zeta \in J(f)$ and a sequence $\left(n_{j}\right)$ with $n_{j} \rightarrow \infty$ as $j \rightarrow \infty$ such that

$$
\left|f^{n}(\zeta)\right| \geq a_{n}, \text { for } n \geq 0, \text { and }\left|f^{n_{j}}(\zeta)\right| \leq a_{n_{j}}^{1+\varepsilon}, \text { for } j \in \mathbb{N} .
$$

If, in addition,

$$
\text { for each } \ell \in \mathbb{N} \text { there exists } n(\ell) \in \mathbb{N} \text { such that } a_{n(\ell)+\ell}<M^{n(\ell)}(R),
$$

then there are uncountably many itineraries with respect to $\left\{A_{n}(R)\right\}$ that correspond to points $\zeta$ satisfying (6.11).

Proof. First we choose $R=R(f, \varepsilon)$ so large that $R \geq \max \left\{R_{0}, R_{2}, R_{3}\right\}$, where $R_{0}=$ $R_{0}(f)$ is the constant in Lemma 2.1 $R_{2}=R_{2}(f)$ is the constant in Corollary 2.3 and $R_{3}=R_{3}(f)$ is the constant in Lemma 4.1, and also so that

$$
M(r)>r^{10000} \text { and } \sqrt{\log r} \geq 100 / \varepsilon, \text { for } r \geq R .
$$

Suppose that $\left(a_{n}\right)$ is any sequence satisfying (6.10). As in the proof of Theorem 6.1. the idea is to choose a suitable sequence of closed annuli $E_{n}$, related to the sequence $\left(a_{n}\right)$, and then apply Lemma 5.1 . 
First define $r_{0}=a_{1}$. By Lemma 4.1, there exists $N(1) \in \mathbb{N}$ and annuli

$$
A_{n}=A\left(r_{n}, r_{n}^{k_{n}}\right), \quad n=0, \ldots, N(1),
$$

with the properties given in that lemma. In particular,

$$
r_{n+1}=M\left(r_{n}\right), \text { for } n=0, \ldots, N(1)-1,
$$

so, by (6.10),

$$
r_{n} \geq a_{n}, \text { for } n=0, \ldots, N(1) .
$$

Also, with

$$
E_{n}=\bar{A}_{n}, \text { for } n=0, \ldots, N(1)
$$

we have

$$
f\left(E_{n}\right) \supset E_{n+1}, \text { for } n=0, \ldots, N(1)-1,
$$

and, for any $S, S^{\prime}, T, T^{\prime}$ that satisfy

$$
2<S<S^{\prime}, \quad T<T^{\prime} \leq M\left(\frac{1}{3} r_{N(1)}^{1+\delta_{N(1)}}\right) \text { and } S^{\prime} \leq \frac{1}{2} T,
$$

we have

$$
f\left(E_{N(1)}\right) \text { contains } \bar{A}\left(S, S^{\prime}\right) \text { or } \bar{A}\left(T, T^{\prime}\right) .
$$

We now apply the covering property (6.18) with

$$
S=a_{N(1)+1} \text { and } S^{\prime}=S^{1+20 \delta(S)}
$$

and

$$
T=S^{1+40 \delta(S)} \text { and } T^{\prime}=T^{1+20 \delta(T)} .
$$

To see that this choice of $S, S^{\prime}, T, T^{\prime}$ is possible first note that, by (6.10), (6.13) and (6.19),

$$
\frac{T}{S^{\prime}}=S^{20 \delta(S)}=\exp (20 \sqrt{\log S}) \geq 2 .
$$

Then, by using (6.19) and (6.20), together with the fact that

$$
S=a_{N(1)+1} \leq M\left(a_{N(1)}\right) \leq M\left(r_{N(1)}\right),
$$

which follows from (6.10) and (6.14), we deduce that

$$
\begin{aligned}
T^{\prime} & =S^{(1+40 \delta(S))(1+20 \delta(T))} \\
& \leq S^{(1+40 \delta(S))(1+20 \delta(S))} \\
& \leq M\left(r_{N(1)}\right)\left(1+40 \delta\left(M\left(r_{N(1)}\right)\right)\left(1+20 \delta\left(M\left(r_{N(1)}\right)\right)\right)\right.
\end{aligned}
$$

The final inequality here holds because the function

$$
t \mapsto t^{(1+40 / \sqrt{\log t})(1+20 / \sqrt{\log t})} \text { is increasing on }[1, \infty) .
$$

Since $\delta\left(M\left(r_{N(1)}\right)\right) \leq \delta_{N(1)} / 100 \leq 1 / 80$, by (6.13), we deduce that

$$
\left(1+40 \delta\left(M\left(r_{N(1)}\right)\right)\right)\left(1+20 \delta\left(M\left(r_{N(1)}\right)\right)\right) \leq 1+\frac{4}{5} \delta_{N(1)},
$$

so

$$
T^{\prime} \leq M\left(r_{N(1)}\right)^{1+\frac{4}{5} \delta_{N(1)}} .
$$

As in the proof of (4.29), it now follows, by Lemma 2.1 part (b) and the fact that $\sqrt{\log r_{N(1)}} \geq 5 \log 3$, that

$$
T^{\prime} \leq M\left(r_{N(1)}\right)^{1+\frac{4}{5} \delta_{N(1)}} \leq M\left(r_{N(1)}^{1+\frac{4}{5} \delta_{N(1)}}\right) \leq M\left(\frac{1}{3} r_{N(1)}^{1+\delta_{N(1)}}\right) .
$$


Thus (6.17) holds with this choice of $S, S^{\prime}, T, T^{\prime}$, so (6.18) does also. Moreover,

$$
a_{N(1)+1}=S<T^{\prime}=S^{(1+40 \delta(S))(1+20 \delta(T))} \leq a_{N(1)+1}^{1+\varepsilon},
$$

by (6.19), 6.20), 6.10) and 6.13).

Hence if we define the pair $\left(r_{N(1)+1}, \delta_{N(1)+1}\right)$ to be either $(S, \delta(S))$ or $(T, \delta(T))$, and put

$$
E_{N(1)+1}=\bar{A}\left(r_{N(1)+1}, r_{N(1)+1}^{k_{N(1)+1}}\right)
$$

where

$$
k_{N(1)+1}=1+20 \delta_{N(1)+1}=1+20 \delta\left(r_{N(1)+1}\right),
$$

then, by (6.18),

$$
f\left(E_{N(1)}\right) \supset E_{N(1)+1},
$$

and, by 6.21),

$$
E_{N(1)+1} \subset \bar{A}\left(a_{N(1)+1}, a_{N(1)+1}^{1+\varepsilon}\right) .
$$

Now, since $E_{N(1)+1}$ has the form in (6.22), we can apply Lemma 4.1 with $r_{0}$ replaced by $r_{N(1)+1}$ to give $N(2) \in \mathbb{N}$ with $N(2)>N(1)$ and closed annuli

$$
E_{n}=\bar{A}\left(r_{n}, r_{n}^{k_{n}}\right), \quad n=N(1)+1, \ldots, N(2),
$$

which satisfy

$$
\begin{gathered}
r_{n} \geq a_{n}, \text { for } n=N(1)+1, \ldots, N(2), \\
f\left(E_{n}\right) \supset E_{n+1}, \text { for } n=N(1)+1, \ldots, N(2)-1, \\
f\left(E_{N(2)}\right) \supset \bar{A}\left(r_{N(2)+1}, r_{N(2)+1}^{k_{N(2)+1}}\right)=E_{N(2)+1}, \text { say, }
\end{gathered}
$$

where

$$
k_{N(2)+1}=1+20 \delta_{N(2)+1}=1+20 \delta\left(r_{N(2)+1}\right)
$$

and

$$
E_{N(2)+1} \subset \bar{A}\left(a_{N(2)+1}, a_{N(2)+1}^{1+\varepsilon}\right) .
$$

Repeating this application of Lemma 4.1 infinitely often, we obtain closed annuli

$$
E_{n}=\bar{A}\left(r_{n}, r_{n}^{k_{n}}\right), \quad n \geq 0,
$$

such that

$$
\begin{gathered}
r_{n} \geq a_{n}, \quad \text { for } n \geq 0, \\
f\left(E_{n}\right) \supset E_{n+1}, \quad n \geq 0,
\end{gathered}
$$

and a strictly increasing sequence of positive integers $N(j), j \in \mathbb{N}$, such that

$$
E_{N(j)+1} \subset \bar{A}\left(a_{N(j)+1}, a_{N(j)+1}^{1+\varepsilon}\right), \text { for } j \in \mathbb{N} .
$$

Since $f$ has no multiply connected Fatou components, all the components of $J(f)$ are unbounded, as noted earlier, so the closed annuli $E_{n}$ must all meet $J(f)$ by (6.26) and the fact that $J(f)$ is completely invariant under $f$.

By (6.26) and Lemma 5.1, there exists $\zeta$ with the property that

$$
f^{n}(\zeta) \in E_{n} \cap J(f), \text { for } n \geq 0 .
$$

By (6.25) and (6.27), this $\zeta$ satisfies (6.11) with $n_{j}=N(j)+1, j \in \mathbb{N}$. 
To prove the final part of Theorem 6.2 we argue in a similar way to the proof of the final part of Theorem 6.1. As in that proof, we deduce from (6.12) that for all $\ell \in \mathbb{N}$,

$$
a_{n+\ell}<M^{n}(R), \text { for } n \geq n(\ell) .
$$

Next note that the covering property (6.18) can be used to show that, for any $j \in \mathbb{N}$,

$$
f\left(E_{N(j)}\right) \supset \bar{A}\left(R^{\prime},\left(R^{\prime}\right)^{k^{\prime}}\right),
$$

where $R^{\prime}$ and $k^{\prime}$ satisfy

$$
R^{\prime} \geq M\left(r_{N(j)}\right) \text { and } k^{\prime}=1+20 \delta\left(R^{\prime}\right) .
$$

The argument required here is similar to that used in the proof of Theorem 4.2 to show that $f\left(B_{N(j)}\right)$ contains an annulus with these properties, and we omit the details.

Therefore in the above proof, for any $j \in \mathbb{N}$, instead of defining the annulus $E_{N(j)+1}$ by choosing the pair $\left(r_{N(j)+1}, \delta_{N(j)+1}\right)$ to be either $(S, \delta(S))$ or $(T, \delta(T))$, where the values of $S$ and $T$ are as given in (6.19) and (6.20) (with the suffix $N(1)+1$ replaced by $N(j)+1)$, we can alternatively define

$$
E_{N(j)+1}=\bar{A}\left(r_{N(j)+1}, r_{N(j)+1}^{k_{N(j)+1}}\right),
$$

where

$$
r_{N(j)+1}=R^{\prime} \geq M\left(r_{N(j)}\right) \text { and } k_{N(j)+1}=k^{\prime}=1+20 \delta\left(r_{N(j)+1}\right) .
$$

Then, by (6.29) and (6.30), we have $f\left(E_{N(j)}\right) \supset E_{N(j)+1}$, and we can then continue the construction as before to obtain the itinerary of a point $\zeta$ that satisfies (6.11).

In view of (6.28), as well as (6.31), 6.32) and (6.10), there must be infinitely many values of $j$ for which these two possible choices of $E_{N(j)+1}$ lie in different elements of the partition $\left\{A_{n}(R)\right\}$. If we now consider all possible ways of choosing $E_{N(j)+1}$ that correspond to a point $\zeta$ satisfying (6.11), then the itineraries with respect to $\left\{A_{n}(R)\right\}$ that arise form the branches of an infinite binary tree and so are uncountable. This completes the proof of Theorem 6.2.

\section{ACKNOWLEDGEMENT}

The authors are grateful to Mitsu Shishikura for helpful comments.

\section{REFERENCES}

[1] I. N. Baker, Wandering domains in the iteration of entire functions, Proc. London Math. Soc. (3) 49 (1984), no. 3, 563-576, DOI 10.1112/plms/s3-49.3.563. MR759304 (86d:58066)

[2] Walter Bergweiler, Iteration of meromorphic functions, Bull. Amer. Math. Soc. (N.S.) 29 (1993), no. 2, 151-188, DOI 10.1090/S0273-0979-1993-00432-4. MR.1216719 (94c:30033)

[3] Walter Bergweiler and A. Hinkkanen, On semiconjugation of entire functions, Math. Proc. Cambridge Philos. Soc. 126 (1999), no. 3, 565-574, DOI 10.1017/S0305004198003387. MR.1684251 (2000c:37057)

[4] W. Bergweiler, P. J. Rippon, and G. M. Stallard, Multiply connected wandering domains of entire functions, Proc. London Math. Soc. (3) 107 (2013), no. 6, 1261-1301. MR3149847

[5] Lennart Carleson and Theodore W. Gamelin, Complex dynamics, Universitext: Tracts in Mathematics, Springer-Verlag, New York, 1993. MR.1230383 (94h:30033)

[6] Robert L. Devaney and Folkert Tangerman, Dynamics of entire functions near the essential singularity, Ergodic Theory Dynam. Systems 6 (1986), no. 4, 489-503, DOI 10.1017/S0143385700003655. MR873428(88e:58057) 
[7] A. È. Erëmenko, On the iteration of entire functions, Dynamical systems and ergodic theory (Warsaw, 1986), Banach Center Publ., vol. 23, PWN, Warsaw, 1989, pp. 339-345. MR.1102727 (92c:30027)

[8] A. Ė. Erëmenko and M. Yu. Lyubich, Dynamical properties of some classes of entire functions (English, with English and French summaries), Ann. Inst. Fourier (Grenoble) 42 (1992), no. 4, 989-1020. MR1196102 (93k:30034)

[9] W. K. Hayman, Subharmonic functions. Vol. 2, London Mathematical Society Monographs, vol. 20, Academic Press Inc. [Harcourt Brace Jovanovich Publishers], London, 1989. MR.1049148 (91f:31001)

[10] M. E. Herring, Mapping properties of Fatou components, Ann. Acad. Sci. Fenn. Math. 23 (1998), no. 2, 263-274. MR.1642181 (2000b:30032)

[11] Masashi Kisaka, On the connectivity of Julia sets of transcendental entire functions, Ergodic Theory Dynam. Systems 18 (1998), no. 1, 189-205, DOI 10.1017/S0143385798097570. MR.1609471(99a:30033)

[12] J. W. Osborne, The structure of spider's web fast escaping sets, Bull. Lond. Math. Soc. 44 (2012), no. 3, 503-519, DOI 10.1112/blms/bdr112. MR2966997

[13] P. J. Rippon and G. M. Stallard, Functions of small growth with no unbounded Fatou components, J. Anal. Math. 108 (2009), 61-86, DOI 10.1007/s11854-009-0018-z. MR2544754 (2011b:30070)

[14] P. J. Rippon and G. M. Stallard, Slow escaping points of meromorphic functions, Trans. Amer. Math. Soc. 363 (2011), no. 8, 4171-4201, DOI 10.1090/S0002-9947-2011-05158-5. MR.2792984(2012d:37108)

[15] P. J. Rippon and G. M. Stallard, Fast escaping points of entire functions, Proc. Lond. Math. Soc. (3) 105 (2012), no. 4, 787-820, DOI 10.1112/plms/pds001. MR2989804

[16] P. J. Rippon and G. M. Stallard, Regularity and fast escaping points of entire functions, Int. Math. Res. Not., DOI 10.1093/imrn/rnt111(2013).

[17] Günter Rottenfusser, Johannes Rückert, Lasse Rempe, and Dierk Schleicher, Dynamic rays of bounded-type entire functions, Ann. of Math. (2) 173 (2011), no. 1, 77-125, DOI 10.4007/annals.2011.173.1.3. MR2753600(2012b:37121)

[18] Jian-Hua Zheng, On uniformly perfect boundary of stable domains in iteration of meromorphic functions. II, Math. Proc. Cambridge Philos. Soc. 132 (2002), no. 3, 531-544, DOI 10.1017/S0305004101005813. MR/1891688(2003b:37069)

Department of Mathematics and Statistics, The Open University, Walton Hall, MilTON Keynes MK7 6AA, United KInGDOM

E-mail address: p.j.rippon@open.ac.uk

Department of Mathematics and Statistics, The Open University, Walton Hall, MilTON Keynes MK7 6AA, United Kingdom

E-mail address: g.m.stallard@open.ac.uk 